\title{
Les bénéfices du télétravail
}

Mobilité modérée : réduction du stress et des émissions de gaz à effets de serre

\section{Patrice Tissandier et Sophie Mariani-Rousset}

\section{CpenEdition}

Journals

Édition électronique

URL : http://journals.openedition.org/rfst/397

DOI : $10.4000 /$ rfst.397

ISSN : 2492-3672

Éditeur

Espaces et SOciétés (UMR 6590)

Référence électronique

Patrice Tissandier et Sophie Mariani-Rousset, «Les bénéfices du télétravail », Revue francophone sur la santé et les territoires [En ligne], Mobilités Transports et Santé, mis en ligne le 27 mai 2019, consulté le 06 avril 2021. URL : http://journals.openedition.org/rfst/397; DOI : https://doi.org/10.4000/rfst.397

Ce document a été généré automatiquement le 6 avril 2021.

\section{(c) (i) (2)(2)}

La Revue francophone sur la santé et les territoires est mise à disposition selon les termes de la Licence Creative Commons Attribution - Pas d'Utilisation Commerciale - Partage dans les Mêmes Conditions 4.0 International. 


\section{Les bénéfices du télétravail}

Mobilité modérée : réduction du stress et des émissions de gaz à effets de serre

Patrice Tissandier et Sophie Mariani-Rousset

\section{Introduction : l'ambivalence du télétravail ?}

Depuis 2008, plus de la moitié de la population mondiale habite un espace urbain (O.N.U., 2008a), un taux de 80\% étant prédit pour 2025 dans les pays développés (O.N.U., 2008b). De cette concentration des hommes et de leurs activités, les zones urbaines apparaissent dès lors comme les espaces à privilégier pour conduire des actions visant à réduire les émissions de gaz à effet de serre (GES). Avec $29 \%$ du total, soit 126,5 millions de tonnes équivalent $\mathrm{CO}$, le transport routier fait partie des principaux émetteurs de GES en France (Commissariat général au développement durable, 2018). Au sein de ce secteur, les navettes domicile-travail couplées à l'utilisation massive de la voiture ont été identifiées comme l'un des plus grands contributeurs (EEA, 2012; EMEP/EEA, 2013 ; CITEPA, 2013).

Le télétravail autorise, de manière volontaire, une activité professionnelle en dehors du lieu principal, grâce aux technologies de l'information et de la communication. Le concept est apparu aux États-Unis en 1976 par l'intermédiaire de la société ATT (Craipeau, 2009-2010). Entendu ici comme la possibilité pour un salarié de travailler depuis son domicile ou un tiers-lieu, permettant une certaine flexibilité (Taskin \& Vendramin, 2005; Morel-à-L'Huissier, 2006), le télétravail est une des solutions possibles aux objectifs de réduction du nombre et/ou de la distance des navettes domicile-travail. Il peut donc être considéré comme un outil de réduction des émissions de GES issues du trafic routier, à la condition que sa mise en place, en bouleversant les boucles de déplacements quotidiens, ne produise pas de nouveaux déplacements.

3 La prise en compte de l'impact du télétravail sur la santé, autant physique que psychique, est tout aussi importante (Baugé, 1994 ; Rey, Sitnikoff, 2006 ; Morin, Gagné, Cherré, 2009). En effet, si des points négatifs sont évoqués dans la littérature comme un potentiel manque de régulations (Taskin, 2006), une source d'envahissement de la vie 
privée (Tremblay, 2006) ou une difficulté à percevoir la frontière vie privée/ professionnelle (Dumas \& Ruiller, 2014), de nombreuses études vantent également la qualité de vie induite par ce nouveau mode de travail : réduction du stress et de la fatigue, meilleurs rapports professionnels et familiaux, moindres déplacements, efficacité dans le travail, santé améliorée (Tremblay \& Le Bot, 2001 ; Vandenberghe \& Delobbe, 2002 ; Bérard et al ., 2002 ; Taskin, 2003 ; Moriset, 2004; D’Amours, 2005 ; Morel-à-L'Huissier, 2006; Tremblay, 2006; Ottmann \& Felio, 2016; Scaillerez \& Tremblay, 2016 ; Taskin, 2016).

4 Le télétravail a ainsi une position ambivalente: à la fois réducteur des navettes domicile-travail, mais possible créateur de nouveaux déplacements; source d'une meilleure qualité de vie en réduisant la fatigue et le stress, mais potentiellement envahisseur de la sphère privée. Fruit d'un financement PREDIT, le projet EFFETS a permis de mener une recherche en régions lilloise et bisontine entre 2010 et 2012. Elle a consisté en une phase d'expérimentation de télétravail au domicile et de suivi longitudinal des actifs concernés pour des entreprises volontaires, et une phase de simulation des mobilités suivant différents scénarii via Mobisim, plateforme de simulation des mobilités quotidiennes et résidentielles développée au laboratoire ThéMA. Le suivi régulier des télétravailleurs lors de la phase d'expérimentation a permis de mettre en évidence une modification des pratiques de mobilité de la part des télétravailleurs, qui a été progressivement introduite dans les simulations afin de les rendre les plus proches possible de la réalité.

5 Les conclusions de ce projet permettent ainsi de répondre à un double objectif. D'une part, il s'agissait de mesurer le ressenti des télétravailleurs (vis-à-vis de leur travail, mais aussi de leur famille) afin d'éviter les dérives et prévenir les risques (d'isolement, de stress, de mal-être lié à l'absence de contrôle) et appréhender les modifications engendrées par cette nouvelle manière de travailler (sur leur santé, leur bien-être, mais aussi sur le mode de fonctionnement de leur cellule familiale). D'autre part, il s'agissait de quantifier l'impact du télétravail sur les déplacements et les niveaux d'émission des gaz à effet de serre (GES) et évaluer les conséquences sur les boucles de déplacements quotidiens en l'absence de structuration par la navette domicile-travail ainsi que sur les modes de déplacement.

\section{EXPÉRIMENTATION DANS DEUX ENTREPRISES ET SIMULATIONS}

6 Afin de répondre aux objectifs de l'étude, un travail préliminaire de recherche bibliographique a mis en évidence certains facteurs essentiels à prendre en compte lors du suivi de télétravailleurs : temps de travail, temps de transport, coût lié au transport vs temps " gagné », actions inhabituelles réalisées, ressenti des télétravailleurs...

7 Pour mesurer ces critères et leur évolution potentielle au cours de l'expérimentation, un suivi longitudinal des télétravailleurs a été réalisé via des entretiens/enquêtes durant la phase d'expérimentation. 


\section{Expérimentation : questionnaires de suivi des télétravailleurs au domicile}

8 La construction et l'administration des questionnaires aux télétravailleurs ont été menés par une équipe interdisciplinaire ${ }^{1}$ qui a regroupé des géographes spécialistes en modélisation des mobilités quotidiennes, des socio-psychologues pour la bonne conduite et l'analyse des sondages et des professionnels de la communication pour la relation avec le télétravailleur et l'employeur. Les buts de ces questionnaires étaient en effet multiples puisqu'il s'agissait autant d'évaluer le gain réalisé par les entreprises et les salariés via l'optimisation des déplacements et l'occupation des locaux (gains de temps, déplacements minimisés, réduction du stress...), que d'obtenir des informations sur les habitudes de déplacement en vue de les introduire par la suite dans les scénarii de simulation.

Deux méthodes d'enquête ont été utilisées : des questionnaires et des entretiens. Deux entreprises pilotes, au fonctionnement différent, ont accepté de mener l'étude, dans la ville de Lille : Okaïdi et Norpac. Ces deux entreprises sont volontaires - ce qui montre à la fois l'intérêt des salariés et la volonté des employeurs d'investiguer de nouvelles méthodes de travail. Le temps d'expérience a été respectivement de 5 et 7 mois. L'enquête a concerné 18 personnes au final, qui ont répondu à un ensemble de 335 questionnaires (avant, pendant et après expérimentation). Le choix du jour de la semaine a été limité (pas le mercredi, pas les jours de réunions, etc.). Certains testeurs sont «à la carte »: ils prennent (ou non) un jour dans la semaine, pas forcément le même, en fonction de leurs besoins et en accord avec leur manager.

10 Du fait des nombreux objectifs, les questionnaires construits sont assez longs puisqu'ils abordent les aspects à la fois :

- sociaux : parle-t-on de freins ou de gains pour l'entreprise?

- écologiques : cela produit-il une baisse des pratiques de mobilité et/ou des comportements différents?

- psychologiques : les travailleurs à distance voient-ils une amélioration de leurs conditions de travail/de vie, font-ils des économies, sont-ils sujets à moqueries, leur bien-être s'améliore-t-il, comment se passent les relations avec les collègues, leur rendement est-il différent, etc. ?

11 L'administration des questionnaires auprès des entreprises et des télétravailleurs s'est faite en fonction de l'avancement de la phase d'expérimentation : avant, pendant et après (tableau 1). 
Tableau 1 : Les trois phases d'expérimentation Simulation des mobilités quotidiennes

\begin{tabular}{|c|c|c|}
\hline $\begin{array}{l}\text { Avant } \\
\text { Deux questionnaires } \\
\text { sont administrés en } \\
\text { une seule fois, l'un à } \\
\text { destination des } \\
\text { travailleurs à distance, } \\
\text { l'autre auprès de } \\
\text { l'employeur ou de son } \\
\text { référent. L'objectif est } \\
\text { de déterminer les } \\
\text { attentes, les } \\
\text { motivations et les } \\
\text { craintes de chacun. Les } \\
\text { résultats sont pris en } \\
\text { compte dans la } \\
\text { construction du } \\
\text { questionnaire } \\
\text { administré pendant la } \\
\text { phase de télétravail. }\end{array}$ & $\begin{array}{l}\text { Pendant } \\
\text { C'est la phase de suivi des pilotes. On compte deux } \\
\text { questionnaires distincts : } \\
\text { L'un est administré lors de chaque journée de } \\
\text { télétravail ou après une période de } 2 \text { jours } \\
\text { successifs de télétravail. Il comprend des prises de } \\
\text { notes personnelles, un journal de bord avec des } \\
\text { questions sur l'humeur du jour, l'environnement } \\
\text { professionnel et familial, l'entente avec les } \\
\text { collègues (possibilité d'inscrire une anecdote), un } \\
\text { journal des déplacements rempli le jour du } \\
\text { télétravail pour connaître les déplacements et leurs } \\
\text { motifs, le kilométrage parcouru et le mode de } \\
\text { transport ; } \\
\text { l'autre interroge, une fois par mois, les sentiments } \\
\text { d'isolement, la charge de travail, le ressenti en } \\
\text { termes de productivité et de comparaison entre les } \\
\text { journées avec et sans travail à distance. L'objectif } \\
\text { est ici de construire une courbe de } \\
\text { satisfaction / insatisfaction, à comparer avec les } \\
\text { attentes et les inquiétudes de départ. }\end{array}$ & $\begin{array}{l}\text { Après } \\
\text { Des entretiens-bilan } \\
\text { avec les personnes } \\
\text { ayant mené } \\
\text { l'expérience closent } \\
\text { l'expérimentation : } \\
\text { utilisation habituelle } \\
\text { des moyens de } \\
\text { transport, évolution } \\
\text { des impressions au fil } \\
\text { de l'expérience, mise } \\
\text { en évidence des } \\
\text { stratégies des } \\
\text { enquêtés pour } \\
\text { résoudre les } \\
\text { problèmes ou les } \\
\text { éviter, solutions } \\
\text { proposées. }\end{array}$ \\
\hline
\end{tabular}

12 MobiSim, plateforme de simulation des mobilités quotidiennes et résidentielles développée par le laboratoire ThéMA de l'Université de Franche-Comté depuis 2008, a été utilisée pour cette étude. MobiSim se situe dans la lignée des Land Use and Transport Integrated (LUTI) Models, au même titre que PROPOLIS, URBANSIM ou MIRO (Antoni, 2010).

13 Deux étapes préliminaires sont nécessaires à la réalisation des simulations. Tout d'abord, du fait des législations nationales et de la protection de la vie privée, les données sont rarement accessibles à l'échelle des individus. En France par exemple, l'information est agrégée par groupes de plusieurs centaines de personnes, pour que les habitants ne puissent être ni isolés ni identifiés (CNIL). Pour pallier cette difficulté, une solution consiste à générer une population dite synthétique (Banos \& Parrott, 2009). Concrètement, il s'agit de générer une population artificielle en se basant sur des données connues au niveau agrégé $n+1$ du recensement, à laquelle sont attribuées des caractéristiques individuelles. Trois raisons justifient cette construction: i) une compatibilité avec les données françaises; ii) une reproductibilité pour toutes les agglomérations ; iii) une échelle fine pour la prise en compte des déplacements courts. La seconde étape consiste à la création du " grid ", soit la reproduction cartographique de l'aire d'étude, essentiellement par l'insertion de données provenant de l'Institut Géographique National (IGN) auxquelles sont notamment ajoutées des informations sur les réseaux de transports (lignes de bus, tram, etc., avec les arrêts et les horaires de passage).

Une fois la population synthétique créée et l'aire d'étude reproduite, MobiSim effectue une distribution des flux de déplacement, et ce via deux étapes: la génération du programme d'activités des individus, dont les télétravailleurs (quel agent ?, quelle activité ?, à quelle heure et dans quel ordre ?) et la distribution des déplacements (d'où partent-ils?, vers quels lieux?, par où passent-ils?). Ces deux étapes ne sont pas indépendantes $\mathrm{du}$ temps: elles sont calculées pour un jour ouvré standard et se succèdent au cours de cette journée selon une approche d'optimisation. 
15 La distribution des mobilités se fonde sur un modèle gravitaire type Huff (1964). Pour chaque motif de déplacement, on cherche à obtenir le nombre d'agents se déplaçant d'un lieu $i$ vers un autre lieu $j$ (figure 1 ).

Figure 1 : Modèle gravitaire type Huff

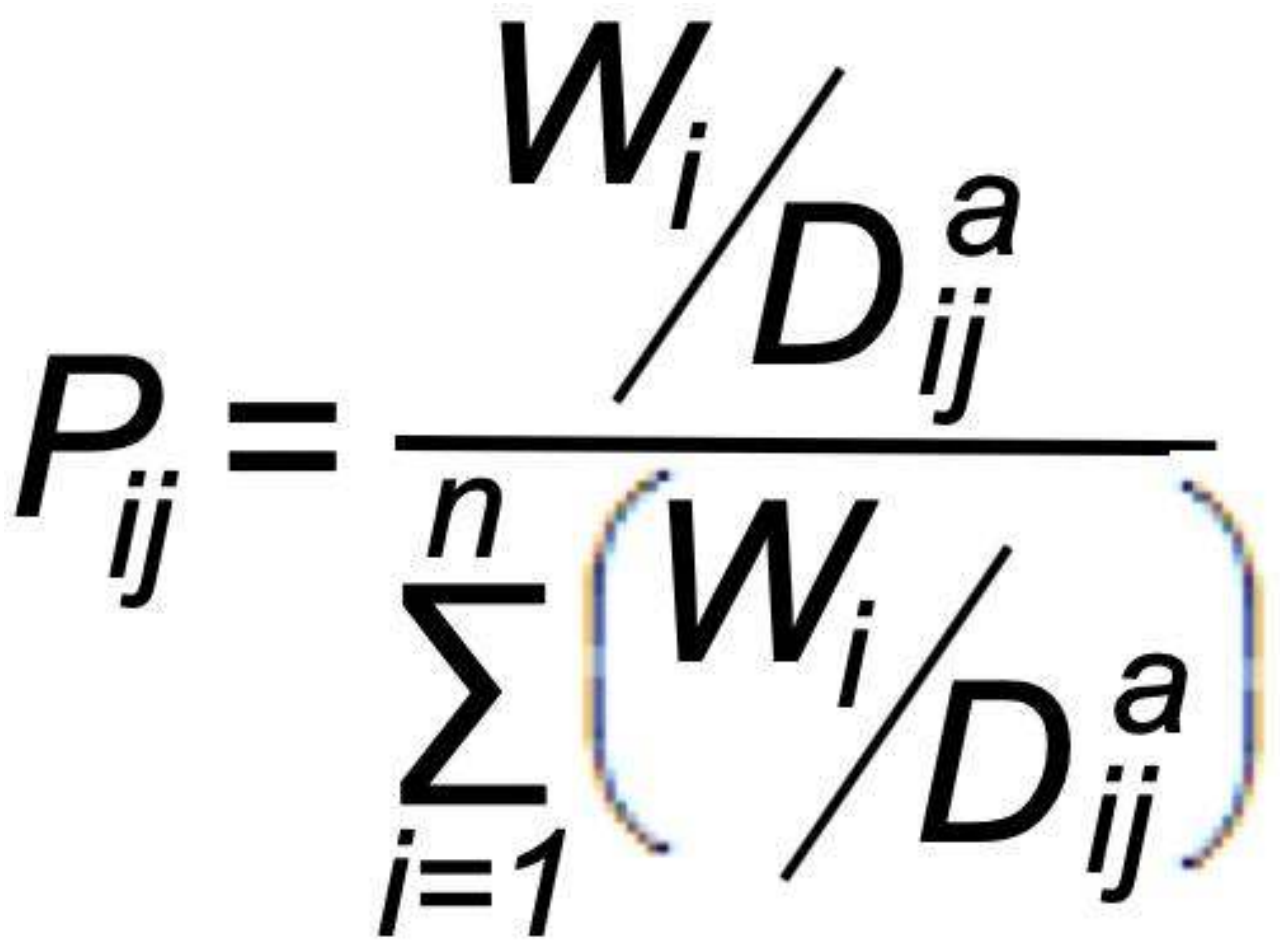

Ce nombre est déduit de la probabilité d'effectuer un déplacement, elle-même obtenue par un modèle gravitaire qui repose sur trois postulats de base :

- la complémentarité : interaction entre l'ensemble des lieux que l'on peut considérer comme complémentaires (lieux de résidence, d'emploi, tiers-lieux sont considérés comme tels);

- la friction de la distance: l'interaction se fonde sur une minimisation, possiblement pondérée, des distances entre les espaces considérés comme complémentaires ;

- l'absence de substitution: pour évaluer les possibilités d'interaction entre les lieux, il est nécessaire de tester toutes les possibilités de complémentarité et distance.

17 Ainsi, les opérations d'affectation sur les réseaux et de calcul d'itinéraires basées sur l'algorithme de Dijkstra impliquent deux postulats. D'une part, l'optimisation des parcours qui amène les individus à prendre nécessairement le plus court chemin et d'autre part, l'omniscience des agents qui connaissent a priori toutes les possibilités de déplacement et tous les chemins.

\section{Construction des scénarii}

À partir de la définition du télétravail donnée en introduction, plusieurs scénarios ont été imaginés, avec un taux plus ou moins important de télétravailleurs, et avec un nombre de tiers- lieux potentiels plus ou moins nombreux. Dans tous les scénarios testés, les télétravailleurs restent à leur domicile ou se rendent dans un tiers-lieux au lieu de se rendre sur leur lieu habituel de travail. Le reste de leur mobilité est également affecté : ils continuent à faire les mêmes activités qu'un jour de travail 
classique, mais peuvent les faire depuis des lieux différents, et s'y rendre avec un mode de transport qui n'est pas celui qu'ils utilisent le reste de la semaine. Les retours obtenus lors de la phase d'expérimentation en matière de modification des pratiques de mobilité (maintien ou suppression de certains déplacements liés à la navette domiciletravail, apparition de nouvelles pratiques) ont par la suite été progressivement incorporés aux phases de simulations pour les rendre le plus proche possible de la réalité.

19 Un travail préliminaire d'indentification et d'inclusion de lieux potentiels de télétravail, en plus du domicile, constitue la première démarche. Les tiers-lieux pouvant potentiellement accueillir des télétravailleurs ont été définis selon trois catégories : les tiers-lieux de type 1, 2 et 3 (tableau 2).

Tableau 2 : Les tiers-lieux du télétravail

\begin{tabular}{|c|c|c|}
\hline $\begin{array}{l}\text { Tiers-lieux de } \\
\text { type 1, déjà } \\
\text { existants et prévus } \\
\text { pour le télétravail : } \\
\text { télécentres, } \\
\text { espaces de } \\
\text { coworking, centres } \\
\text { d'affaires, situés } \\
\text { essentiellement } \\
\text { dans le centre des } \\
\text { agglomérations. }\end{array}$ & $\begin{array}{l}\text { Tiers-lieux de type 2, déjà } \\
\text { existants, utilisés par les } \\
\text { télétravailleurs nomades, } \\
\text { mais non prévus à cet effet : } \\
\text { l'ensemble des lieux } \\
\text { disposant d'une connexion } \\
\text { wifi (cafés, restaurants, } \\
\text { hôtels, gares, parcs publics, } \\
\text { médiathèques...), situés } \\
\text { plutôt en centre-ville, mais } \\
\text { également dans les } \\
\text { banlieues et quelques } \\
\text { communes périurbaines. }\end{array}$ & $\begin{array}{l}\text { Tiers-lieux de type 3, lieux identifiés comme } \\
\text { pouvant accueillir des télétravailleurs dans un } \\
\text { avenir plus ou moins proche, pour répondre à la } \\
\text { demande de nouveaux télétravailleurs } \\
\text { potentiels : mairies et autres administrations } \\
\text { (hôtels de région et de département), agences } \\
\text { Pôle Emploi, centres sociaux ou médico-sociaux. } \\
\text { Les bureaux de poste, dont le cœur de métier } \\
\text { est la communication, pourraient également } \\
\text { proposer des places en complément des autres } \\
\text { lieux, si leur situation géographique ou les } \\
\text { besoins de la population le nécessitent. } \\
\text { L'addition de ces tiers-lieux permet d'offrir au } \\
\text { moins une possibilité de télétravail dans chaque } \\
\text { commune. }\end{array}$ \\
\hline
\end{tabular}

20 Une fois les lieux potentiels de télétravail, en plus du domicile, identifiés et localisés, plusieurs simulations avec des taux de télétravailleurs différents ont ensuite été menées. Ces différents taux de télétravailleurs émanent de résultats d'études sur la thématique du télétravail.

21 En premier lieu, un rapport du Centre d'Analyse Stratégique (CAS, 2009) souligne que le potentiel de télétravailleurs n'est pas uniforme pour toutes les régions. Une méthode de calcul afin d'obtenir une estimation régionale en fonction des potentiels par métiers a été construite sur cette base. Cette estimation repose sur un croisement entre des informations contenues dans les Déclarations Annuelles des Données Sociales (fichiers DADS) indiquant la nature de l'emploi (durée, condition d'emploi, rémunération), les caractéristiques des salariés (sexe, âge, qualification) et des données sur les établissements employeurs (secteur d'activité, taille, emploi présentiel ou non). Les résultats montrent que la région Nord-Pas de Calais, dont Lille est la préfecture, se place en 4 ème position (sur les 22 régions françaises au moment de l'étude) en nombre potentiel de télétravailleurs avec un taux de $21 \%$ pour 2008. En appliquant le même taux de progression entre 2008 et 2015 que celui indiqué dans l'étude, le potentiel serait de 35\% en région Nord-Pas de Calais en 2015. En Franche-Comté (pour Besançon), le potentiel est plus faible puisque le potentiel estimé de télétravailleurs se situe à $16,6 \%$ des salariés; avec un taux de croissance similaire, ce potentiel s'accroît à $27,6 \%$ pour 2015.

Dans un second temps, le nombre de télétravailleurs est également déterminé à partir des statistiques de la DARES de 2004, selon lesquelles 5,4\% des actifs télétravaillent en 
dehors de leur domicile au moins une fois dans la semaine. Ce taux est ventilé par catégorie socio-professionnelle : ce sont les ingénieurs et cadres qui télétravaillent le plus (près de $30 \%$ ), suivis des professions intermédiaires (11\%), des employés (4\%) et des ouvriers (1\%). Par conséquent, le nombre de télétravailleurs dans une commune dépend du nombre d'actifs, mais également de leur profession.

La combinaison des résultats de ces études et des potentiels qui en découlent, déclinés par communes et par catégories socio- professionnelles, ont permis d'implémenter au sein de MobiSim un scénario "plausible » caractérisé par un taux de télétravail fixé à $11 \%$ et 2 jours par semaine pour chaque zone d'étude, mais avec des distinguos selon les types d'emplois et les communes (entre $6 \%$ et $47 \%$ des actifs, une ou deux fois par semaine, selon la CSP et le type d'emploi).

24 Afin de mesurer l'impact d'une politique publique forte en faveur du télétravail, deux scenarii ayant pour caractéristiques des taux extrêmement élevés de télétravailleurs ont été testés. Un premier scénario dit « haut » concerne la possibilité de télétravailler pour $50 \%$ des cadres, $30 \%$ des professions intermédiaires et $20 \%$ des employés à raison de 2 jours par semaine et $10 \%$ des ouvriers une fois par semaine. Le second, dit «très haut » s'applique à $75 \%$ des cadres, dont $25 \%$ une fois par semaine et $50 \%$ deux fois par semaine ; $60 \%$ des professions intermédiaires, avec $20 \%$ une fois par semaine et $40 \%$ deux fois par semaine ; $45 \%$ des employés, dont $15 \%$ une fois par semaine et $30 \%$ deux fois par semaine ; et $20 \%$ des ouvriers, $10 \%$ à une fois par semaine, $10 \%$ à deux fois.

Afin d'obtenir un étalon, une simulation en situation initiale, soit sans télétravail, s'appuyant en partie sur les résultats des enquêtes ménages-déplacements disponibles sur les deux aires d'étude, est réalisée. Le nombre de déplacements quotidiens est alors de 3237346 pour Lille Métropole et de 516809 pour la Communauté d'Agglomération du Grand Besançon.

26 La répartition selon les modes de transport confirme la prédominance du véhicule particulier avec $54,9 \%$ des déplacements à Lille Métropole et $61,4 \%$ dans la Communauté d'Agglomération du Grand Besançon. Les résultats pour la marche à pied sont proches pour les deux territoires $(34,4 \%$ du total des déplacements à Lille et $31,4 \%$ à Besançon). En conséquence, les transports en commun sont davantage utilisés à Lille (10,7\% des déplacements) qu'à Besançon $(7,2 \%)$.

27 La distance moyenne d'un déplacement est plus élevée à Lille avec $4,46 \mathrm{~km}$ contre $3,86 \mathrm{~km}$ à Besançon, du fait de la différence de superficie des aires urbaines concernées. En déclinant selon le mode de transport, si la distance moyenne d'un déplacement avec un véhicule particulier est proche à Lille et Besançon $(5,0 \mathrm{~km}$ et $5,4 \mathrm{~km})$ elle est plus importante tant pour les transports en commun que pour la marche à pied à Lille (respectivement 10,0 km et 1,8 km contre 4,6 km et 0,6 km pour Besançon).

$28 \mathrm{Au}$ final, les déplacements quotidiens génèrent près de $14400000 \mathrm{~km}$ à Lille, dont 8,9 millions en voiture particulière, et près de 2 millions de $\mathrm{km}$ à Besançon dont 1,7 millions en voiture particulière (tableau 3 ). 
Tableau 3 : Résultats des simulations sur les deux territoires d'étude sans télétravail

\begin{tabular}{|l|l|c|c|}
\hline \multicolumn{2}{|l|}{} & Lille & Besançon \\
\hline Nombre total de déplacements quotidiens & 3237346 & 516804 \\
\hline \multirow{3}{*}{ Parts modales (\%) } & MAP & 34,4 & 31,4 \\
\cline { 2 - 4 } & TC & 10,6 & 7,2 \\
\cline { 2 - 4 } & VP & 54,9 & 61,4 \\
\hline \multirow{3}{*}{$\begin{array}{l}\text { Distances } \\
\text { moyennes (km) }\end{array}$} & MAP & 1,80 & 0,64 \\
\cline { 2 - 4 } & TC & 10,00 & 4,61 \\
\cline { 2 - 4 } & VP & 5,00 & 5,42 \\
\cline { 2 - 4 } & \multicolumn{1}{|c|}{ Moyenne } & 4,46 & 3,86 \\
\hline \multirow{3}{*}{$\begin{array}{l}\text { Distances totales } \\
\text { (km) }\end{array}$} & MAP & 2081551 & 104011 \\
\cline { 2 - 4 } & TC & 3466533 & 170902 \\
\cline { 2 - 4 } & VP & 14438774 & 1721444 \\
\cline { 2 - 4 } & \multicolumn{1}{c|}{ Total } & 1996357 \\
\hline
\end{tabular}

MAP : marche à pied ; TC : transport en commun ; VP : véhicule particulier

\section{Des résultats encourageants}

\section{Retour sur expérience : la santé primordiale}

Différents enseignements ont émergé de la phase d'expérimentation menée sur le territoire lillois. Parmi ces enseignements, certains en relation avec une modification des pratiques de mobilité ont été inclus dans les simulations comme cela sera présenté par la suite (Tissandier \& Mariani-Rousset, 2013).

\section{Sentiment d'isolement : des stratégies mises en place}

Parmi les éléments de vigilance évoqués lors du suivi, l'isolement est le plus souvent cité. Il est parfois décrit comme " pesant » lorsqu'une question a besoin d'une réponse rapide.

« Le télétravail c'est bien quand les outils fonctionnent, sinon on se trouve seul face

à son problème, et là c'est l'isolement total !!! "

31 Toutefois, cette sensation engendre la mise en place de plusieurs stratégies chez les testeurs : certains vont utiliser les mails et le téléphone plus qu'à la normale, d'autres regroupent leurs questions et soit les envoient par mail, soit les évoquent le lendemain au bureau. Globalement, plus d'un ou deux jours de télétravail par semaine pourraient engendrer un sentiment d'isolement et des difficultés de communication : on voit ici la place essentielle de la «communication informelle» que le travail au domicile restreint. Cette conclusion est partagée par la moitié des testeurs - qui estime que le rythme d'une journée de télétravail par semaine est adapté - et par un télétravailleur qui, en réalisant deux jours de télétravail par semaine, évoque un seuil à ne pas dépasser.

La communication est essentielle pour contrer ce sentiment d'isolement, et ce également bien avant la mise en place du télétravail. En effet, le suivi a permis de mettre en exergue la nécessité de communiquer les modalités du télétravail auprès de l'équipe en amont. Seulement un tiers des enquêtés avait organisé une réunion d'information auprès de leur équipe, les autres regrettant de ne pas avoir assez formalisé les éléments de l'expérimentation. Les testeurs soulignent donc qu'il faut 
bien insister sur la communication afin de ne pas créer de confusions et que ce mode de fonctionnement soit bien intégré par l'entourage professionnel.

33 Une analyse croisée entre les questionnaires de suivi longitudinal (à la semaine et au mois) et les entretiens-bilan montre que la communication reste le facteur le plus ambigu : dans les réponses fournies, la plupart des enquêtés ne ressentent ni un plus grand besoin de communiquer ni un sentiment d'isolement quand ils travaillent à la maison. Grâce au mail, au téléphone et au communicator (logiciel de partage d'outils) il n'est pas difficile de contacter ses collègues. Néanmoins, lors de certaines périodes où il est nécessaire de communiquer davantage, le télétravail peut être problématique sans communication en face à face. Ces points d'isolement et de manque de communication ressortent dans les entretiens comme les principaux inconvénients de ce type de fonctionnement pour $1 / 3$ des enquêtés.

\section{Réduction du stress et effets sur la santé} adapté à leur posture) - voire une amélioration de leurs problèmes médicaux.

«[Le télétravail] minimise mes douleurs dorsales. Je n'ai donc pas à lutter contre cette douleur pendant 5 jours d'affilée, ce qui me permet de travailler de façon optimale »; « Des problèmes de santé m'obligent à rester à la maison récemment. Le fait de pouvoir travailler depuis la maison est très appréciable ».

Le télétravail agit positivement sur deux éléments source de stress: l'absence de déplacement (pas de retard le matin au travail, ni le soir pour par exemple récupérer les enfants) et la prise de recul par rapport à la multitude des interactions professionnelles (collègues ou clients pressés, réunions impromptues...). Travailler chez soi occasionne une plus grande souplesse et permet de prendre des pauses plus nombreuses, à différents moments de la journée, sans regard / jugement extérieur.

Le fait de ne pas être au contact direct avec ses collègues permet de conserver une certaine distance lors d'interactions perturbantes.

"Le e-travail permet des soupapes "; "En pilotant à distance des sujets de crise, j'avais la capacité à ne pas être impacté par le stress des autres; j'ai donc pu agir comme une tour de contrôle"; "Stress beaucoup moins important lors de la journée de télétravail, car travail au calme, sans être tout le temps dérangé, et beaucoup moins de bruit qu'au travail en open space ».

Concernant la santé, les enquêtés évoquent un plus grand confort physique (matériel

apparaît cependant que des difficultés potentiellement stressantes durant une journée de télétravail sont évoquées lors de problèmes techniques et informatiques. Concernant ces problèmes, plusieurs propositions sont faites comme la mise en place d'une messagerie instantanée, la réalisation de conférences téléphoniques ou encore l'utilisation de la Webcam pour entrer en contact avec ses collègues. Une hotline avec un professionnel s'occupant spécifiquement des télétravailleurs et pouvant intervenir en cas de problème sérieux est également une solution.

Malgré ce point de vigilance, il ressort que près de $80 \%$ des enquêtés ont constaté une baisse du stress et de la fatigue.

\section{Relations avec l'entreprise et les collègues}

La mise en place du télétravail a également eu pour conséquence une amélioration de l'image de leur entreprise, et ce pour 15 testeurs (sur 18). Pour 1/4 d'entre eux, la mise 
en place du télétravail est une marque de confiance; pour les autres, le télétravail accorde à l'entreprise une certaine ouverture d'esprit et est signe d'innovation et de progrès social. La notion de confiance apparaît assez souvent au cours des entretiens et semble être importante pour les enquêtés. Il ressort des entretiens que le télétravail est un mode de fonctionnement moderne qui va dans le sens de l'évolution du monde du travail et qui s'adapte aux contraintes des professionnels. En cas d'offre d'emploi dans une autre entreprise, le télétravail pourrait jouer en la faveur de l'entreprise.

Pour la moitié des enquêtés, il n'y a pas de soucis particuliers avec les clients puisque les télétravailleurs sont joignables par mail et par téléphone. La plupart d'entre eux procèdent à un transfert d'appels. Pour un tiers des interrogés, le télétravail ne nécessite pas d'adaptation particulière vis-à-vis des clients. Il faut juste savoir anticiper, gérer son travail et ne pas oublier à la maison les documents nécessaires. Les relations interpersonnelles au sein de l'équipe du télétravailleur semblent peu impactées: les télétravailleurs ne ressentent pas de jugements de la part de leurs collègues. Certains sont simplement curieux et posent des questions. Malgré cela, plusieurs enquêtés ont le sentiment de devoir se justifier, de prouver qu'ils travaillent autant qu'au bureau. Pour une grande majorité des enquêtés, le lien avec l'équipe et le manager est resté stable.

«J'ai communiqué efficacement avec mes collègues afin de préparer la journée du lendemain. »

\section{Productivité améliorée}

41 Toutes les personnes interrogées soulignent les effets positifs du télétravail sur leur productivité. Dans les questions ouvertes, quelques enquêtés précisent que ce n'est pas tant la charge de travail qui a augmenté, mais que le télétravail engendre une organisation différente qui permet un gain en rapidité et en quantité de travail. Cette productivité plus importante peut donner l'impression d'avoir plus de travail alors que l'efficacité n'est pas forcément liée à la charge de travail en tant que telle. Un testeur précise qu'il est normal de plus travailler les jours de télétravail puisque c'est une façon de compenser le gain de temps et le confort qu'apporte ce mode de fonctionnement.

" Je suis de plus en plus impatiente d'être au mardi pour profiter de cette journée à avancer sur des sujets et être moins dérangée, donc plus productive. Cette journée représente pour moi un énorme gain en confort!».

42 Certains managers sont surpris par l'avancée des télétravailleurs, plus productifs. Même lorsqu'il y a hésitation (11\% des réponses ont été " oui et non ", mais jamais «non ${ }^{2}{ }^{2}$ ), les effets bénéfiques du télétravail sur leur bien-être et leur productivité sont présents - notamment dans le fait de travailler au calme et de ne pas être sans cesse coupé dans les activités par les collègues, le bruit, etc. (pas d' open space avec 200 personnes). Les enquêtés mettent en avant, dans un premier temps, l'aspect quantitatif : ils gagnent en rapidité $(44,4 \%)$, en efficacité $(44,4 \%)$ et en quantité de travail $(27,8 \%)$. C'est l'occasion pour certains de rattraper du retard, de travailler sur des dossiers de fond, des tâches complexes et des travaux d'analyse. À temps identique, ils travaillent davantage chez eux qu'au bureau. $22,2 \%$ des interrogés spécifient que grâce au télétravail ils gagnent en concentration. Ceci est le plus souvent lié au fait de travailler au calme et de ne pas être dérangé dans son activité. effectuer des tâches complexes, à traiter des "gros dossiers ». Bien que les enquêtés 
affirment que leur productivité est meilleure, aucun ne dispose d'indicateurs objectifs permettant de mesurer cette amélioration, sauf ceux dont le manager a remarqué que les dossiers avançaient mieux les jours de télétravail. Les enquêtés insistent beaucoup plus sur le gain de temps que sur la qualité du travail effectué : 44,4\% d'entre eux précisent que le télétravail permet d'être plus rapide.

\section{Qualité de vie et vie familiale}

Une amélioration de leur qualité de vie est également soulignée par la grande majorité des télétravailleurs.

"C'est agréable de porter une tenue plus décontractée pour travailler. Aujourd'hui

j'ai travaillé pieds nus et par cette chaleur c'était plutôt bienvenu. »

Le travail au calme engendre une baisse de stress.

« Pause goûter avec un cornet de glace sur la terrasse, au soleil, dans un transat... ça

détend bien!»

Le temps gagné en restant à la maison est réinvesti dans les tâches quotidiennes - ce qui permet un gain de temps sur la journée et une meilleure organisation générale. Par ailleurs, les enquêtés indiquent qu'il y a une meilleure conciliation vie personnelle / vie professionnelle: ils peuvent davantage profiter de leur famille (par exemple, accompagner les enfants à l'école, déjeuner avec leur compagnon...) ou prendre des rendez-vous personnels en fin de journée, libérant ainsi le samedi matin ou le fait de devoir poser une demi-journée de congés. Ensuite, c'est tout l'aspect utile et confortable du télétravail qui est mis en avant. Une grande partie des enquêtés $(77,4 \%)$ indique que leur entourage familial a très bien réagi à leur passage en télétravail. Certains ont fixé les règles dès le début afin de ne pas créer de confusions; d'autres expliquent que leur famille apprécie de passer plus de temps en leur compagnie. Il apparaît également que les pauses sont principalement réinvesties en tâches ménagères. Plusieurs testeurs précisent qu'ils font un peu de ménage et de rangement pendant que l'ordinateur s'allume. D'autres profitent du temps gagné et de la pause de midi pour les loisirs (sport, amis...).

«Comme je fais du sport entre 12 et $14 \mathrm{~h}$, je suis en tenue décontractée toute la

matinée »;

«Quelques coups de fils pour RV personnels [dans la journée] »;

«Quelques minutes le matin, consacrés aux artisans »

À noter que le réinvestissement en temps de travail peut amener à une amplitude horaire assez importante. Le plus souvent entre 30 minutes et 2 heures de plus que lors d'une journée au bureau classique; mais il arrive que les testeurs travaillent encore plus (parfois jusque tard le soir). La plupart d'entre eux font moins de pauses et certains réduisent le temps de celle de midi. En travaillant sur place, il est plus difficile de se fixer une limite pour arrêter. Peut-être est-ce une des limites du télétravail.

S'il n'y a pas de route à faire, un tiers des enquêtés réinvestit le temps de trajet en sommeil et en travail.

"Je peux dormir une heure de plus »;

«Le gain est surtout sur le sommeil et le temps que je peux consacrer à ma famille

(grâce au temps de transport en moins) ».

Les télétravailleurs dorment plus, mais commencent à la même heure ou plus tôt puisqu'ils sont directement sur place. Ce temps économisé permet surtout de passer plus de temps en famille (22,2\%). 
«J'en ai profité pour emmener et aller chercher les enfants à l'école (...). Déjeuner en famille ";

«RV médical pour mon fils N. »;

« Je conduis mon fils le mardi à l'école, du coup je le lève plus tard et ça se ressent

donc sur son état de fatigue. »

Ainsi, des forces positives et négatives s'exercent sur les télétravailleurs. Les apports positifs sur la santé, nettement plus importants, peuvent être résumés ainsi (figure 2) :

Figure $n^{\circ} 2$ : Apports positifs du télétravail sur la santé

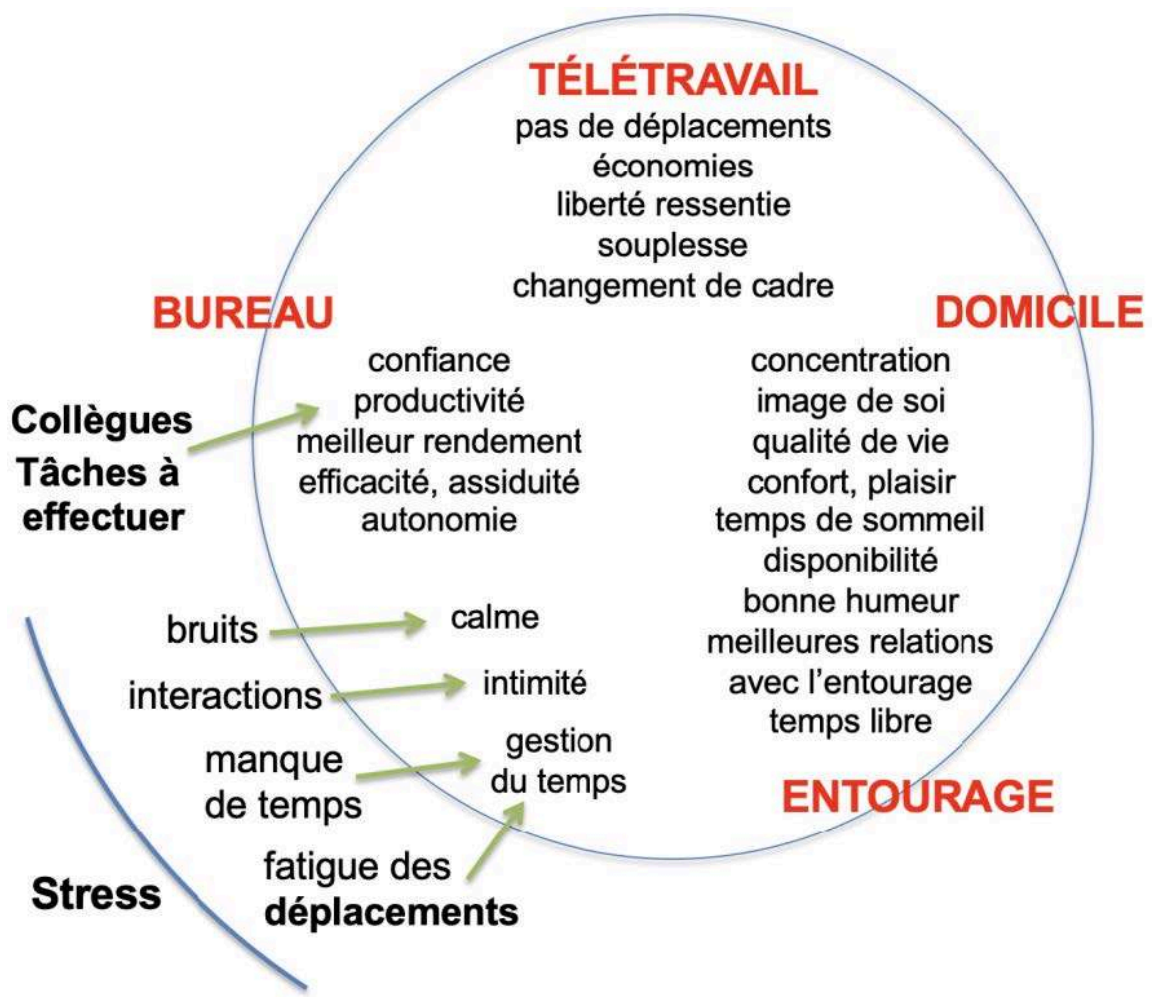

Déplacements : un changement des pratiques de mobilité

Le télétravail engendre principalement un gain en frais de déplacement. Toutefois, ce gain est compensé par une perte en frais énergétiques (chauffage/électricité du domicile) et en frais de repas (absence de tickets restaurant un jour de télétravail). C'est un élément à prendre en compte et à formaliser si une entreprise est amenée à instaurer officiellement le télétravail. Si les gains en frais de transport et la limitation des déplacements représentent une inscription dans une politique de développement durable, cela n'est cependant pas évoqué par les testeurs.

Les questionnaires de suivi longitudinal, notamment par l'intermédiaire du journal quotidien des déplacements, fournissent également des informations importantes sur les pratiques de déplacements. La grande majorité des testeurs utilise la voiture pour aller au travail et ce, principalement, pour des raisons pratiques. En effet, ils précisent que l'utilisation des transports en commun constitue une réelle perte de temps sans compter ceux dont le lieu d'habitat est mal desservi et où l'utilisation des transports en commun est quasiment impossible. À noter aussi qu'aucun des enquêtés ne pratique le covoiturage, là aussi, pour des raisons pratiques. 
Néanmoins, les résultats permettent d'observer que le télétravail limite véritablement les trajets : la moitié des enquêtés fait moins de déplacements le jour où ils travaillent à la maison et 7 (sur 18) ne se déplacent pas du tout. Si ceci peut sembler bénéfique d'un point de vue écologique, le sujet n'est aucunement abordé par les testeurs au cours des entretiens. Ces différentes données ont par la suite été implémentées aux modèles de simulation.

«Avec la densité du trafic routier, l'heure d'arrivée peut s'avérer être source de stress (retard occasionné par accident, intempérie...). Le salarié subi, alors qu'en télétravail (...) le salarié est libéré de cette inquiétude et débute sa journée sereinement, cela a un impact non négligeable sur sa qualité de vie au travail. »

\section{La simulation et l'impact sur la mobilité}

54 Les résultats présentés par la suite résultent d'un taux de télétravail identique fixé à $11 \%$ des actifs, mais pour 3 scénarii différents : i) le scénario 1 correspond à l'ouverture des tiers-lieux de type 1 ; ii) le scénario 2 à la présence des tiers-lieux de type 1 et 2 ; iii) le scénario 3 à la possibilité de télétravailler dans tous les lieux listés en type 1, 2 et 3 . Ces scénarii, conduits en parallèle de la phase d'expérimentation, ont été modifiés en fonction des retours de suivi des télétravailleurs afin d'introduire une pondération dans certains algorithmes. En effet, pour les télétravailleurs au domicile, l'expérimentation nous a permis de constater le maintien de certains déplacements (comme la dépose des enfants à l'école et leur possible retour certains midis, alors que cela ne se réalisait pas au préalable), voire dans certains cas l'apparition d'activités auparavant réalisées sur d'autres jours (achats essentiellement). Cette modification des pratiques de mobilité a été incluse dans les simulations au moment de la génération du programme d'activités des télétravailleurs.

\section{Augmentation générale de la mobilité}

Les résultats des trois scénarios montrent tous une augmentation générale de la mobilité des individus, qui se matérialise par une croissance du nombre de déplacements, et ce pour les deux territoires d'étude (figure 3). Une des explications réside dans la plus grande proximité entre domicile et lieu de télétravail, notamment lors de l'ouverture des tiers-lieux de type 3 localisés dans toutes les communes, proximité qui semble avoir un impact sur les boucles de déplacements structurées par les pérégrinations domicile-travail.

Figure 3 : Évolution du nombre total de déplacements suivant les scénarii testés
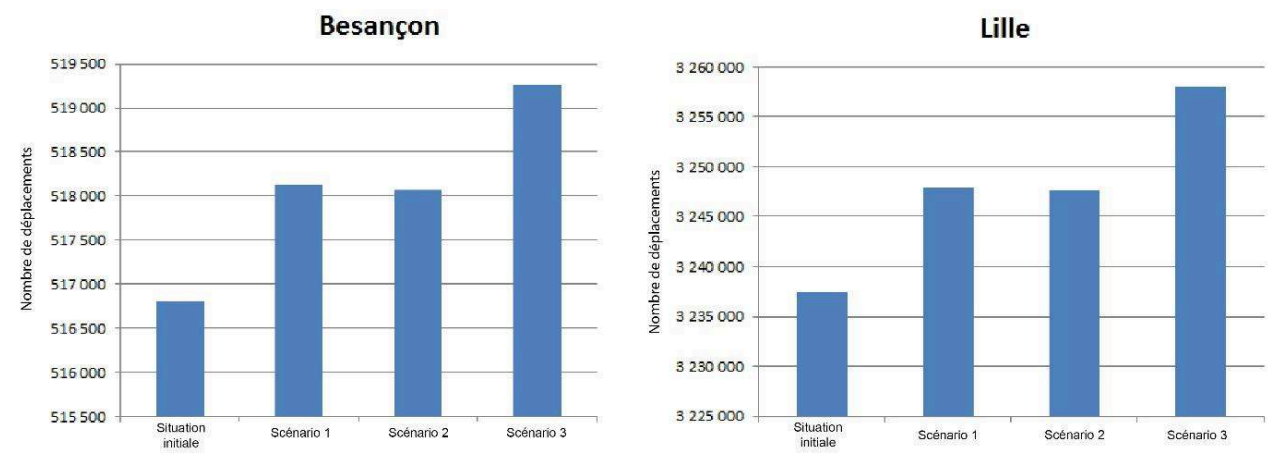
Dans le scénario 1, lors de l'ouverture des tiers-lieux de type 1 (espaces de coworking et centres d'affaires), un peu plus de 1300 déplacements pour Besançon $(+0,26 \%)$ et de 10000 déplacements supplémentaires pour Lille sont générés. Les chiffres sont sensiblement identiques avec l'accès aux tiers-lieux de type 2 (hôtels, cafés, restaurants et lieux publics disposant du Wifi). Cependant, l'ouverture du type 3 (mairies, pôles emploi, bureaux de poste) procure une hausse bien plus importante puisque plus de 20000 déplacements supplémentaires pour Lille $(+0,64 \%$ par rapport à la situation initiale) et près de 2500 pour Besançon $(+0,48 \%)$ viennent s'ajouter. Comme évoqué plus haut, on peut supposer que l'ouverture des tiers-lieux de type 3 provoque une désagrégation des boucles de déplacements et l'apparition de mobilités davantage polarisées par le domicile (retour au domicile à la fin du travail, du fait de sa proximité, avant de repartir pour d'autres activités).

\section{Report modal vers la marche à pied}

Cette croissance du nombre de déplacements se caractérise également par une modification des comportements de mobilité (figure 4) avec un report sur la marche à pied : le nombre de déplacements effectués en voiture particulière se réduit, dans un degré moindre celui des transports en commun, ceci au profit de la marche à pied.

Figure 4 : Évolution des parts modales en nombre de déplacements
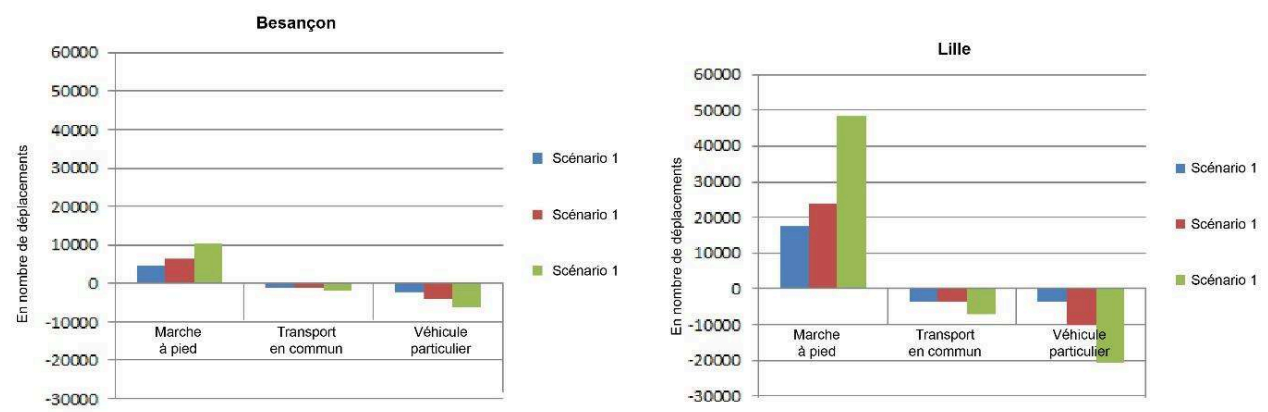

Néanmoins (figure 5), en proportion, la régression de l'utilisation des transports en commun lors de la mise en place du télétravail avec tiers-lieux potentiels est plus importante que pour les véhicules particuliers.

Figure 5 : Évolution en pourcentage des parts modales
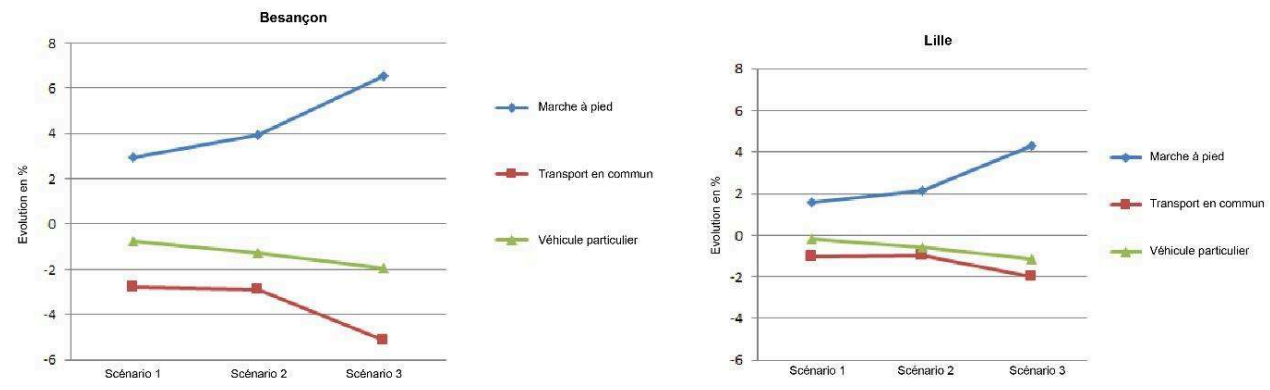

Ces résultats, qui montrent que les effets du télétravail favorisent un accroissement de la pratique de la marche à pied, sont donc en partie contrebalancés par la réduction plus massive de l'utilisation des transports en commun que de la voiture particulière, 
nuançant en conséquence les possibles effets sur la réduction des émissions de GES. De tels résultats peuvent être expliqués par la plus grande proximité entre le lieu de résidence et le lieu d'emploi, notamment lors de l'ouverture des tiers-lieux de type 3 présents dans chaque commune, qui favorise la marche à pied, mais qui conduit en outre à une inadéquation entre l'organisation des réseaux de transports en commun, qui relient le plus souvent la périphérie au centre-ville, et la demande en déplacements « locaux », qui reste internes à la même commune.

\section{Réduction globale des distances parcourues}

Logiquement, si la marche à pied s'accroît aux dépens des autres modes de déplacements, et malgré l'augmentation générale du nombre de déplacements, les distances parcourues quotidiennement au sein des deux territoires d'étude sont en régression (figure 6).

Figure 6 : Évolution des distances parcourues en nombre des kilomètres
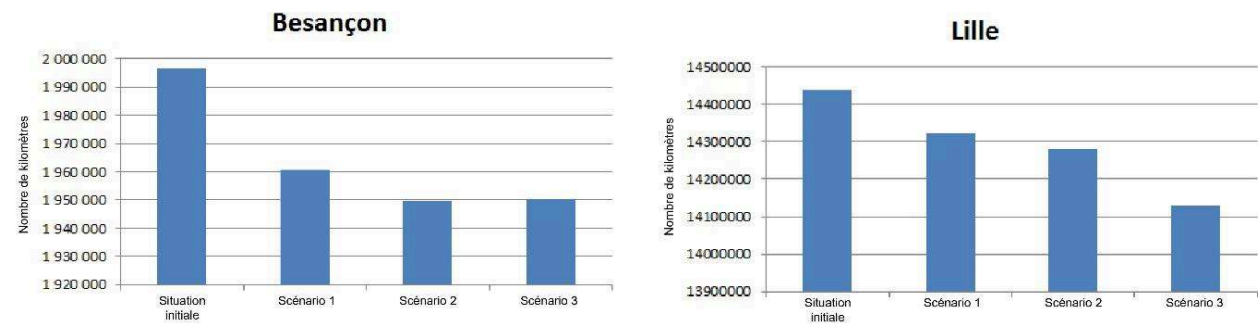

61 En valeur absolue, la réduction des distances parcourues quotidiennement est importante puisque, suivant les scénarios, ce sont entre 35000 et $45000 \mathrm{~km}$ quotidiens qui ne sont pas effectués à Besançon et entre 115000 et $310000 \mathrm{~km}$ pour Lille. Néanmoins, la mise en perspective de ces chiffres avec les distances qui sont parcourues au quotidien rend compte d'un impact plus faible de l'activité télétravail puisque, dans le meilleur des cas, ce sont moins de $2,5 \%$ des distances qui sont annulées.

La faiblesse de cet impact est à mettre également en lien avec les effets sur la part des différents modes de transport dans les distances totales parcourues : malgré l'essor de la marche à pied, ce mode de déplacement ne concerne que des distances faibles et, de facto, la voiture particulière conserve une part prépondérante dans ce total, comme l'illustrent les figures 7 et 8. 
Figure 7 : Évolution en pourcentage des distances parcourues

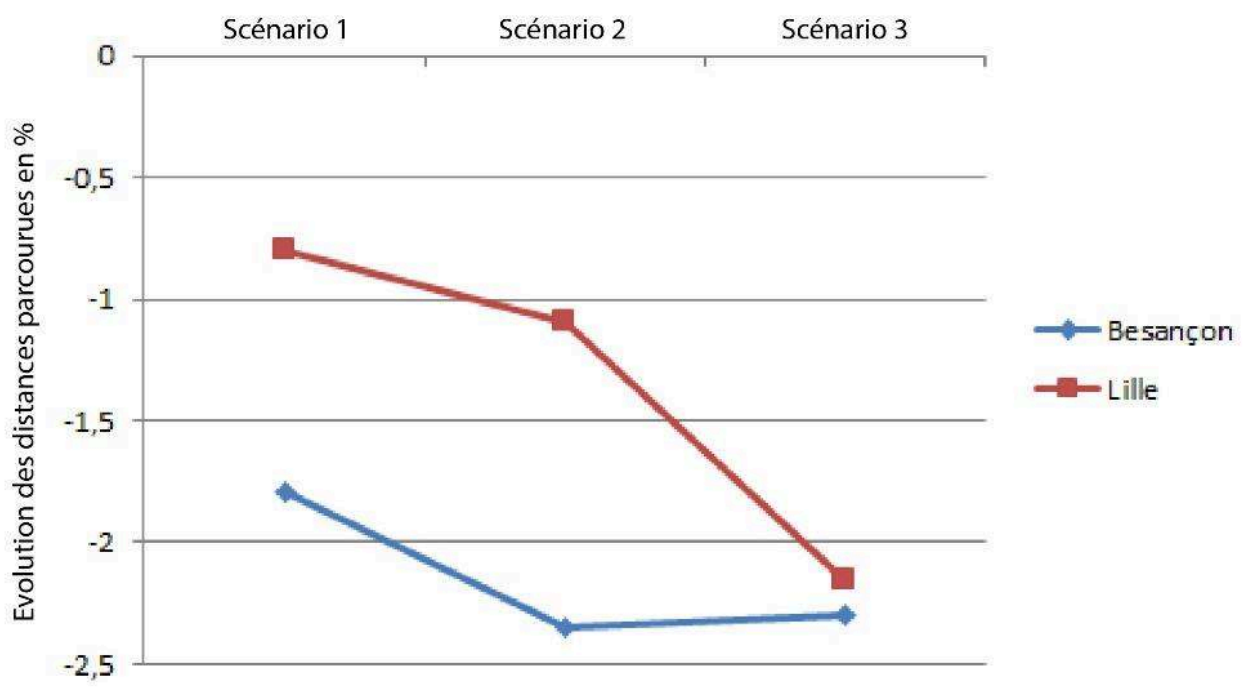

Figure 8 : Évolution des distances parcourues, en km, suivant les modes de transport
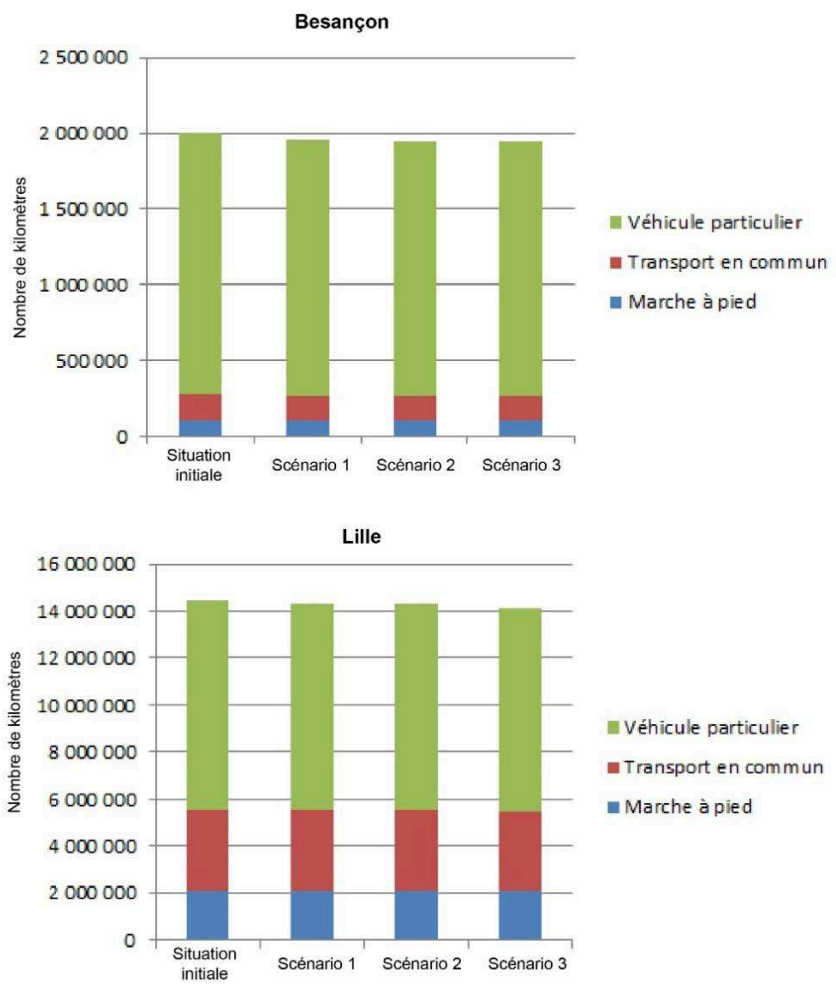

\section{Un scénario utopique : un fort développement du télétravail}

Les 3 scenarii détaillés précédemment avaient pour point commun un taux identique de télétravailleurs, seule l'ouverture de tiers-lieux potentiels les différenciant. Pour compléter cette analyse, nous avons également souhaité mesurer l'impact d'un fort accroissement du télétravail, comme présenté plus haut. 
64 avec la possibilité de télétravailler soit au domicile, soit dans les 3 types de tiers-lieux. Seuls les résultats pour la métropole lilloise sont présentés par la suite.

Figure 9 : Évolution du nombre de déplacements et des distances parcourues selon les scénarii « haut » et « très haut » à Lille.
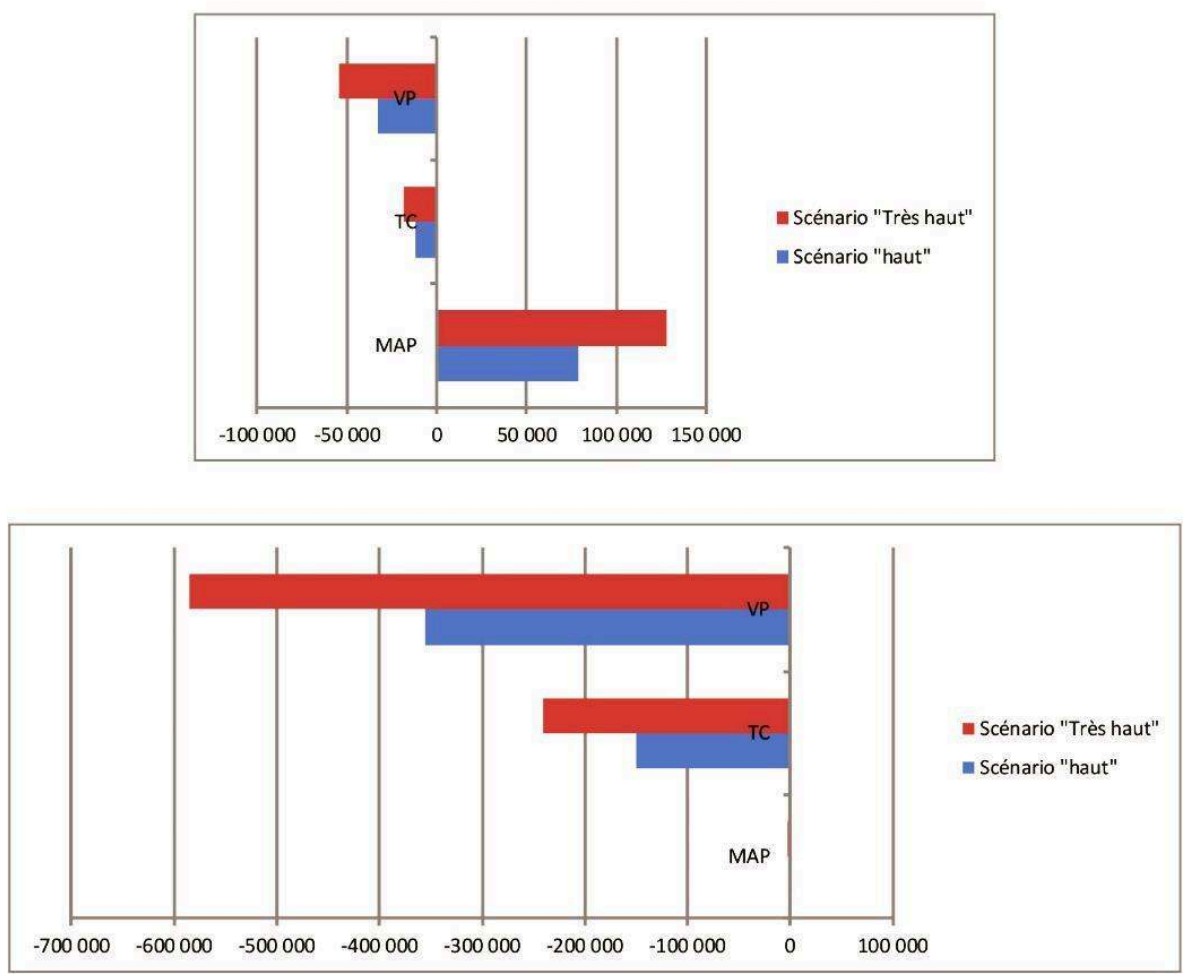

L'analyse des résultats de ces deux simulations montre une similitude avec les conclusions précédentes : augmentation de la mobilité générale caractérisée par une forte croissance, en nombre de déplacements, de la marche à pied et une baisse des transports en commun et de la voiture particulière, réduction du nombre total de kilomètres parcourus avec des valeurs absolues élevées $(-504000 \mathrm{~km}$ en situation "haute» et $-830000 \mathrm{~km}$ en situation "très haute» en cumulant transports en commun et voiture personnelle), mais plus faible en valeur relative: les distances parcourues en voiture particulière baissent de $6,6 \%$ dans le cas du scénario «très haut ", contre près de $7 \%$ pour les transports en commun.

Les résultats de ces scénarii, caractérisés par des taux extrêmement élevés de télétravailleurs qui peuvent paraître utopiques, confirment que le télétravail est un levier permettant d'agir sur les émissions de GES issues du trafic automobile. À ce titre, ces résultats confirment sa place dans les politiques publiques (telles que la loi Grenelle du 3 août 2009 et la loi relative à la transition énergétique pour la croissance verte) qui cherchent à atteindre les objectifs de la COP 21. Cependant, il est évident qu'il ne peut représenter, pris isolément, la solution à la problématique des transports urbains. 


\section{Conclusion : apports bénéfiques... sur participants volontaires}

De manière générale - grâce au gain de temps économisé sur les transports - les enquêtés se sentent plus productifs, moins fatigués, davantage libres; leur entourage professionnel et familial réagit bien quant à leur situation de télétravailleur ; rares sont ceux qui se sentent seuls ou mal encadrés, ou pour le moins trouvent-ils des solutions.

Le suivi longitudinal des télétravailleurs lors de la phase d'expérimentation souligne clairement l'amélioration de la qualité de vie, tant sur le plan matériel (réduction des coûts liés aux déplacements notamment), que physique (moindre fatigue) ou psychologique (moins de stress, amélioration des relations familiales). Ces résultats ont une part d'explication dans le protocole mis en place pour cette expérimentation : en ne recrutant que des actifs volontaires auprès d'entreprises également volontaires et ayant été sensibilisées préalablement à l'expérimentation, le projet a pu bénéficier d'une adhésion totale du télétravailleur et nullement d'une mise en situation forcée. Il s'agit là certainement d'un des critères fondamentaux de réussite. La souplesse du système élaboré et son adaptation aux réalités professionnelles - comme télétravailler lors de certaines phases d'études et ne pas télétravailler quand il y a des événements importants au travail - joue également un rôle crucial. Cette souplesse doit cependant être un point de vigilance : si les télétravailleurs ont apprécié une journée de télétravail par semaine et que certains ont souhaité l'étendre à une deuxième journée, aucun ne désire aller au-delà par crainte de péjorer la qualité relationnelle avec les supérieurs hiérarchiques ou les collègues. D'un point de vue économique, l'expérience se révèle positive pour l'environnement, pour l'entreprise, pour le salarié. Mais toute éventuelle généralisation du procédé ne devra ni se faire au détriment des salariés, ni nuire à terme à la sociabilité des individus : en cas de maladie ou de grève, le salarié n'a pas à être obligé de travailler chez lui. La phase de simulation des mobilités via les différents scénarii montre un impact significatif du télétravail : augmentation générale de la mobilité, mais changement des modes de déplacement avec réduction de la place de l'automobile et des transports en commun au profit de la marche à pied. Ainsi, si la suppression de certaines navettes domicile-travail, structurantes au sein des pratiques de mobilité quotidienne, semble engendrer un moindre usage du véhicule particulier au profit de la marche à pieds, l'utilisation des transports en commun est également en déclin. On peut donc ici souligner une inadéquation plus importante entre l'organisation des réseaux de transport en commun et les besoins de mobilité des télétravailleurs. En outre, au regard des pourcentages de diminution des distances parcourues, le potentiel de réduction des émissions de GES n'est pas discutable, mais est néanmoins à nuancer: un taux élevé de télétravail n'est pas synonyme d'une réduction drastique des émissions de GES. À ce titre, si le télétravail représente un réel potentiel d'action pour la réduction de l'usage de la voiture, il doit cependant être couplé avec d'autres actions afin d'obtenir des effets notables.

69 Au-delà des aspects purement environnementaux, le télétravail pourrait également tenir une place plus importante dans les politiques de santé publique, notamment en valorisant le rôle bénéfique de la marche à pied sur la santé (US Department of Health and Human Services, 1996 ; UK National Health Service, 2000). Ce constat est confirmé par certains télétravailleurs, qui bien que relevant la difficulté d'équilibrer parfois vie 
privée / vie professionnelle, valorisent les bienfaits du télétravail sur la santé physique et mentale.

En ne réalisant pas certains déplacements les jours télétravaillés comme l'ont montré aussi bien la phase d'expérimentation que de simulation, temps qui est réinvesti dans des activités qui peuvent traditionnellement être effectuées sur un autre jour avec les autres membres de la famille (achats notamment), le télétravail a un impact plus global sur le planning d'activité de l'ensemble du ménage et sur des jours non télétravaillés. En conséquence, le télétravail a potentiellement un « effet ricochet », réorganisant les habitudes de mobilité de la cellule familiale. Une étude complémentaire, questionnant les télétravailleurs sur leurs pratiques de mobilité les autres jours de la semaine permettrait de venir combler ce manque de connaissance.

\section{BIBLIOGRAPHIE}

Antoni, J.-P. (2010). Mobisim. Un modèle multi- agents et multiscalaires pour simuler les mobilités urbaines, in Modéliser la ville. Forme urbaine et politiques de transport, Antoni J.-P. (ss. dir.), Paris : Economica, Coll. Méthodes et approches, pp. 50-77.

Banos, A., Parrott, L. (2009). Simulation spatiale à base d'agents, Hermès, Paris.

Baugé, J. (1994). Quelques effets du télétravail sur la santé et la vie quotidienne, Archives des Maladies Professionnelles et de Médecine du Travail, $n^{\circ}$ 55/8, pp. 644-645.

Bérard, D. (coord.), Kouloumdjian, M-F., Fourrier, C., Montarnal, C. (2002). Le télétravail en question. Analyse critique à partir de 10 cas d'entreprises, Études et Documents, coll. ANACT.

CITEPA (2013). Inventaire des émissions de polluants atmosphériques et de gaz à effets de serre en France. Séries sectorielles et Analyses étendues, Centre Interprofessionnel Technique d'Études de la Pollution Atmosphérique, Paris.

Commissariat général au développement durable (2018). Chiffres clés du transport 2018, Datalab. Craipeau, S. (2009-2010), Télétravail : le travail fluide, Quadermi [en ligne], mis en ligne le 5 janvier 2012.

D’Amours, L. (2005). Télétravail : Concilier performance et qualité de vie. Montréal, IQ : CEFRIO.

Dumas, M., Ruiller, C. (2014). Le télétravail : les risques d'un outil de gestion des frontières entre vie personnelle et vie professionnelle ?, Management \& Avenir $n^{\circ}$ 8, pp. 71-95.

EEA (2012). The contribution of transport to air quality. TERM 2012: transport indicators tracking progress towards environmental targets in Europe, European Environment Agency, Luxembourg.

EMEP/EEA (2013). EMEP/EEA air pollutant emission inventory guidebook 2013. Technical guidance to prepare national emission inventories, European Monitoring and Evaluation Programme \& European Environment Agency, Luxembourg.

Huff, D.L. (1964). Defining and Estimating a Trading Area, Journal of Marketing, vol. 28, p. 38.

Morel-à-L'Huissier, P. (2006). Du télétravail au travail mobile : un enjeu de modernisation de l'économie française. Rapport officiel, Paris : La Documentation française. 
Morin, E. M., Gagné, C., Cherré, B. (2009). Donner un sens au travail : promouvoir le bien- être psychologique, Institut de recherche en santé et en sécurité du travail du Québec. http:// www.irsst.qc.ca/publications-et-outils/publication/i/100500/n/donner-un-sens-au-travailpromouvoir-le-bien-etre-psychologique-r-624/redirected/1

Moriset, B. (2004). Télétravail, travail nomade : le territoire et les territorialités face aux nouvelles flexibilités spatio-temporelles du travail et de la production, Cybergeo ${ }^{\circ} 257,6$ fév. 2004. https://journals.openedition.org/cybergeo/3815

O.N.U. (2008a). State of the world's cities 2008/9 - Harmonious cities, United Nations Habitat \& Earthscan, London.

O.N.U. (2008b). World Urbanization Prospects: The 2007 Revision, United Nations Department of Economic and Social Affairs/Population Division, New York.

Ottmann, J-Y., Felio, C. (2016). Qualité de vie du travail indépendant. Journée Brestoise de Recherche en GRH : La qualité de vie au travail. Enjeux et perspectives, juin 2016, Brest. http:// www.univ-brest.fr/laboici/menu/jrb-grh

Rey, C., Sitnikoff, F. (2006), Télétravail à domicile et nouveaux rapports au travail, Revue Interventions économiques [en ligne], $\mathrm{n}^{\circ}$ 34, mis en ligne le 01 juillet 2006.

Scaillerez, A., Tremblay, D-G. (2016). Le télétravail, comme nouveau mode de régulation de la flexibilisation et de l'organisation du travail : analyse et impact du cadre légal européen et nordaméricain, Revue de l'organisation responsable, $\mathrm{n}^{\circ}$ 11/1, pp. 21-31.

Taskin, L. (2003). Les Enjeux du télétravail pour l'organisation, Reflets et Perspectives, XLII, 2/1, 81-94. https://www.cairn.info/revue-reflets-et-perspectives-de-la-vie-economique-2003-1page-81.htm

Taskin, L., Vendramin, P. (2005). Le télétravail, une vague silencieuse : Les enjeux socioéconomiques d'une nouvelle flexibilité, Louvain : Presses universitaires de Louvain.

Taskin, L. (2006). Télétravail : Les enjeux de la déspatialisation pour le management humain. Revue Interventions économiques, $\mathrm{n}{ }^{\circ} 34$ [En ligne], consulté le 21 sept. 2012. http:// interventionseconomiques.revues.org/680

Taskin, L. (2016). New ways of working : Paradoxes et enjeux, Midis de la GRH. http:// hrpublic.be/wp-content/uploads/2016/09/coll_24042015_laurent taskin.pdf

Tissandier, P., Mariani-Rousset, S. (2013). Le télétravail, un outil de mobilité durable pour une ville durable, Colloque ASRDLF, Mons, Belgique, 8-11 juil.

Tremblay, D-G., Le Bot, I. (2001). Le télétravail : articuler qualité de vie et performance. Rapport de recherche, Québec : CEFRIO.

Tremblay, D-G. (dir.) (2006). Télétravail, travail nomade, e-work et travail à domicile : les enjeux actuels, Revue Interventions économiques 34 [En ligne], consulté le 22 sept. 2012. http:// interventionseconomiques.revues.org/680

UK National Health Service (2000). National Service Framework for Coranary Heart Disease, Departement of Health and Social Care, $128 \mathrm{p}$.

US Department of Health and Human Services (1996). Physical Activity and Health, Report of the Surgeon General.

Vandenberghe, Ch., Delobbe, N. (eds) (2002). Bien-être au travail et transformations des organisations, Louvain-la-Neuve : PUL. 


\section{NOTES}

1. Igor Agbossou, Jean-Philippe Antoni, Pierre Frankhauser, Patrice Tissandier (ThéMA), Sophie Mariani-Rousset (ELLIADD/ThéMA); Gaëlle Eudeline, Fanny Oliveira (Flexineo); Faustine Grillo (rapport de synthèse); Michaël Bruniaux, Thomas Röhr (SEM Numerica); Sylvie Mathon (CETE Nord-Picardie), Nadine Bernard (ChronoEnvironnement); Karine Szymanski Pannetier (Lille Métropole); Géraldine Bodard, Christine Bugajny (CETE NP); Quentin Bakhtiari (MEDDTL/PREDIT), Michel Legros (Agence Idaho).

2. Rappelons que les chiffres concernent les résultats des 335 réponses apportées aux questionnaires par les 18 testeurs.

\section{RÉSUMÉS}

Le télétravail consiste en la possibilité de réaliser son activité professionnelle hors des locaux de l'employeur grâce aux technologies de l'information et de la communication. Forme d'organisation du travail à distance engendrant des avantages, mais aussi des contraintes, le télétravail interroge la société dans ses dimensions environnementales, économiques et humaines. Face à ce constat, cet article propose d'étudier l'impact du télétravail sur la qualité de vie des télétravailleurs ainsi que sur la mobilité. Ce projet pluridisciplinaire a reposé sur deux points : le recrutement dans deux entreprises-pilotes puis le suivi longitudinal de télétravailleurs en phase d'expérimentation, qui a permis de saisir l'ensemble des modifications engendrées par cette nouvelle organisation via l'intermédiaire de questionnaires réguliers ; une modélisation des déplacements domicile-travail et leurs simulations couplées à un calcul des pollutions émises en fonction de différents taux de télétravailleurs. Malgré les aspects contraignants, les résultats montrent les conséquences positives dues à l'absence de mobilité - tant sur la santé individuelle (moins de fatigue, meilleure gestion du temps) que collective (moins de pollution et d'usage des infrastructures, gestion des locaux).

Telework allows, on a voluntary basis, a professional activity outside the main workplace, thanks to information and communication technologies.

As a form of distance work organization, teleworking examines society in its environmental, economic and human dimensions (assets and constraints). The EFFETS' project, conducted in France, aims to measure and analyze the impact of distance work - considered here as the possibility for an individual to work from home or from a third place, other than his or her usual place of work, one or twice a week. To do this, two surveys were performed.

Initially, several employees from two different companies participated in an experiment to work at home on certain days for several months. Some of them were "à la carte", that is to say they had a package of ten days they placed as they wanted according to professional requirements, management and colleagues. Others were "regular", that is, they did one to two days of work each week at home. This participation was made on the basis of employee volunteerism with the agreement of the hierarchy. The testers answered an online questionnaire each week and each month; and at the end of the experiment, they participate in an interview to analyze issues about teleworking, especially effects on physical and mental health. 
Two objectives were allocated to this 6-month experimentation phase with 18 people employed in 3 organizations ( 2 private companies, 1 public administration): first, to better understand the feelings of those involved (through interviews) and second, to estimate the impact of change in daily mobility on travel and the reduction of GHG emission levels through a simulation via the MobiSim platform, within the ThéMA laboratory. MobiSim is a Land Use and Transport Integrated (LUTI) model, quite similar to PROPOLIS, URBANSIM or MIRO. Several "plausible" scenarios were thus established, and two scenarios, aimed at measuring the impact of a strong public policy in favor of telework, were tested. A first "high" scenario concerns the possibility of teleworking for $50 \%$ of executives, $30 \%$ of intermediate professions and $20 \%$ of employees at 2 days per week and $10 \%$ of workers once a week in third places (type 1,2 and 3). The second, said "very high" applies to $75 \%$ of executives, of which $25 \%$ once a week and $50 \%$ twice a week; $60 \%$ of the intermediate professions, with $20 \%$ once a week and $40 \%$ twice a week; $45 \%$ of employees, $15 \%$ once a week and $30 \%$ twice a week; and $20 \%$ of workers, $10 \%$ to once a week, $10 \%$ to twice. In order to obtain a standard, a simulated initial situation, without teleworking, is carried out on both study areas.

Simulations and experiments were carried out in the territories of Lille Métropole and the "Communauté d'Agglomération du Grand Besançon"; the investigation took place in Lille. If we note a general increase in mobility, it is however through a change in modes of travel - with reduction of the car use (and public transport) in favor of walking.

The reduced drop in mobility thanks to teleworking shows that, overall, the experiment is successful - both in terms of the effects on pollution and the emission of greenhouse gas and on the physical and moral health of the respondents: positive effects on stress are significant and the quality of life is improved (Figure).

INDEX

Mots-clés : pratiques professionnelles, mobilité, télétravail, stress, pollution de l'air

\section{AUTEURS}

\section{PATRICE TISSANDIER}

Maître de conférences en géographie Université de Franche-Comté, France, laboratoire ThéMA

\section{SOPHIE MARIANI-ROUSSET}

Maître de conférences en psychologie Université de Franche-Comté, France, laboratoire ELLIADD/CCM 